\title{
Experiência emocional de enfermeiros atuantes na linha de frente durante a pandemia de Covid-19
}

\author{
Emotional experience of frontline nurses during the Covid-19 pandemic
}

\author{
Experiencia emocional de las enfermeras de primera línea durante la pandemia de Covid-19
}

\section{Graciela da Silva Migueis ${ }^{1^{*}}$, Michelle Sales da Silva ${ }^{2}$, Renata Aparecida Faria de Araujo ${ }^{3}$, Nivaldo Pereira Filho $^{4}$, Wanmar de Souza Oliveira ${ }^{5}$, Washington Júnio Oliveira ${ }^{6}$}

Como citar esse artigo. Migueis, G. S; da Silva, M. S; de Araújo, R. A. F; Filho, N. P Oliveira, W. S; Oliveira, W. J. Experiência emocional de enfermeiros atuantes na linha de frente durante a pandemia de Covid-19. Revista Pró-UniverSUS. 2021 Jul./Dez.; 12 (2): 70-72.

\section{Resumo}

A pandemia pelo novo coronavirus tem transformado em todos os aspectos a vida das pessoas mundialmente. Muitos profissionais, em especial a enfermagem, precisam lidar continuamente em suas atividades laborais com essa doença nova e desconhecida, tornando-as mais vulneraveis ao adoecimento fisico e emocional. Nesse sentido, o objetivo deste estudo é retratar as condições emocionais dos enfermeiros atuantes frente a pandemia. Será um estudo qualitativo, com entrevista semiestruturada a ser realizada com os enfermeiros atuantes nas unidades de internação destinados a pacientes com Covid-19 em um hospital de referencia no interior de Mato Grosso, Brasil. Espera-se que o estudo possa retratar os sentimentos, medos, anseios dos sujeitos e se tornar instrumento de pesquisa para subsidiar ações de prevenção às doenças emocionais.

Palavras-chave: Enfermeiros; Covid-19; Emoções, Experiência de vida.

\begin{abstract}
The new coronavirus pandemic has transformed the lives of people worldwide in all aspects. Many professionals, especially nurses, need to continually deal with this new and unknown disease in their work activities, making them more vulnerable to physical and emotional illness. In this sense, the aim of this study is to portray the emotional conditions of nurses working in the face of the pandemic. It will be a qualitative study, with semi-structured interviews to be carried out with nurses working in inpatient units for patients with Covid-19 in a reference hospital in the interior of Mato Grosso, Brazil. It is expected that the study can portray the feelings, fears, anxieties of the subjects and become a research instrument to support actions to prevent emotional illnesses.
\end{abstract}

Keywords: Nurses; Covid-19; Emotions; Life experience.

${ }^{1}$ Doutora em Biotecnologia e Biodiversidade. Docente do Curso de graduação em Enfermagem e da pós-graduação em Residência Multiprofissional em Saúde do Adulto e Idoso da Universidade Federal de Rondonópolis. Rondonópolis, Mato Grosso, Brasil. Email: gmigueis@yahoo.com.br. ORCID*: https://orcid.org/0000-0001-7287-6026

${ }^{2}$ Doutora em Recursos Naturais. Docente do Curso de graduação em Enfermagem e da pós-graduação em Residência Multiprofissional em Saúde do Adulto e Idoso da Universidade Federal de de Rondonópolis. Rondonópolis, Mato Grosso, Brasil. Email: michele.salles@ufr.edu.br. ORCID*: https://orcid.org/0000-0002-4076-5628

${ }^{3,4}$ Discentes do primeiro ano da Pós-Graduação em Residência Multiprofissional em Saúde do Adulto e Idoso da Universidade Federal de Rondonópolis. Rondonópolis, Mato Grosso, Brasil. Email: araujore24@gmail.com; nivaldo96@outlook.com. ORCID*: https://orcid.org/0000-0002-1021-5115; https://orcid.org/0000-0002-8279-1808

${ }_{5,6}$ Discentes do segundo ano da Pós-Graduação em Residência Multiprofissional em Saúde do Adulto e Idoso da Universidade Federal de Rondonópolis. Rondonópolis, Mato Grosso, Brasil. Email: w-anmar@hotmail.com; wasj1oliveira@gmail.com ORCID*: https://orcid.org/0000-0002-7233-4777; http://orcid.org/0000-0002-8020-8594.

* Email de correspondencia: gmigueis@yahoo.com.br 


\section{Resumen}

La nueva pandemia de coronavirus ha transformado la vida de las personas en todo el mundo en todos los aspectos. Muchos profesionales, especialmente enfermeros, necesitan lidiar continuamente con esta nueva y desconocida enfermedad en sus actividades laborales, haciéndolos más vulnerables a las enfermedades físicas y emocionales. En este sentido, el objetivo de este estudio es retratar las condiciones emocionales de las enfermeras que trabajan frente a la pandemia. Será un estudio cualitativo, con entrevistas semiestructuradas a realizar con enfermeras que laboran en unidades de internación de pacientes con Covid19 en un hospital de referencia en el interior de Mato Grosso, Brasil. Se espera que el estudio pueda retratar los sentimientos, miedos, ansiedades de los sujetos y convertirse en un instrumento de investigación para apoyar acciones de prevención de enfermedades emocionales.

Palabras clave: Enfermeras; Covid-19; Emociones; Experiencia de vida.

\section{Introdução}

Um novo tipo de coronavírus (Sars-Cov-2) caracterizada pela síndrome respiratória aguda e com potencial altamente infecciosa foi identificada em dezembro de 2019, na cidade de Wuhan, província de Hubei, na República Popular da China, denominada Covid-19. ${ }^{1}$ Sendo altamente contagiosa, se espalhou de forma muito rápida e, no final de janeiro de 2020 fora declarado surto do novo coronavirus. ${ }^{1,2}$

Nesse cenário, a Organização Mundial de Saúde (OMS) declarou Emergência de Saúde Pública de Importância Internacional (ESPII) e no Brasil, foi declarada Emergência de Saúde Pública de Importância Nacional (ESPIN) pelo vírus SARS-CoV-2. Em março, declarou-se pandemia ${ }^{3}$ resultado da velocidade com que o vírus tem se espalhado entre os Países, e dentro de cada um, tem influenciado o cotidiano de bilhões de pessoas no mundo. ${ }^{4}$

O coronavirus tornou-se grande desafio para a saúde pública mundial. Os impactos vivenciados frente a este vírus de rápida propagação na população ocasionaram crescente demanda de internações hospitalares devido agravos respiratórios causados pelo Covid-19 e inclusive com alto número de óbitos. A crescente demanda trouxe também a necessidade de mais recursos humanos para o atendimento à saúde da população. ${ }^{5}$

Com isso, os ambientes de trabalho relacionado a saúde sofreram intensas modificações nas rotinas, requerendo adaptações, fluxo de atendimento a paciente com Covid-19, implementação de protocolos e intensivas capacitações. Os profissionais de saúde, em especial a enfermagem, que estiveram e ainda estão atuando na linha de frente ao enfrentamento do Covid-19, tiveram que se adaptar as mudanças emergentes necessárias perante uma nova situação, ainda desconhecida.

Nessa perspectiva, as equipes de saúde correm mais riscos de contraírem o coronavírus por estarem mais expostos, ${ }^{6}$ e corroborando com o estudo de revisão ${ }^{7}$ que buscou identificar as condições de trabalho dos profissionais de saúde/enfermagem no contexto da pandemia apontou que as condições laborais não são as adequadas e tornou-se mais desfavorável neste momento.

Assim, é evidente que as circunstâncias vividas pelos profissionais de enfermagem atuantes no atendimento a paciente com Covid-19 são potenciais geradores de estresse. Nesse contexto, é essencial a realização de novos estudos que abordem o modo como estes profissionais se sentem, uma vez que, se encontram mais vulneraveis ao adoecimento fisico e emocional. Assim, o objetivo deste estudo é retratar as condições emocionais dos enfermeiros atuantes frente a pandemia.

\section{Metodologia}

A presente nota prévia faz parte de um estudo que será desenvolvido a partir de um projeto matricial no Programa de Residência Multiprofissional em Saúde do Adulto e Idoso.

Trata-se de um estudo de abordagem qualitativa. A pesquisa qualitativa responde a questões muito particulares. Ela se preocupa, nas ciências sociais, com um nível de realidade que não pode ser quantificado. Ou seja, ela trabalha com o universo de significados, motivos, aspirações, crenças, valores e atitudes, o que corresponde a um espaço mais profundo das relações, dos processos e dos fenômenos que não podem ser reduzidos à operacionalização de variáveis. ${ }^{8}$

A pesquisa será realizada em um hospital no interior de Mato Grosso, Brasil. O hospital possui característica predial, é referência para 19 municípios do Estado e atende diversas especialidades. Diante da pandemia instaurada mundialmente, o hospital se tornou referência para o atendimento a pacientes acometidos 
por Covid-19, e assim, alguns setores de assistência ao paciente foram reorganizados para o atendimento prioritário para essa biocenose.

A pesquisa realizar-se-á com todos os enfermeiros que atuam nos setores de atendimento a pacientes internados por Covid-19, sendo três setores. Os setores são: Unidade de Terapia Intensiva Covid, Unidade de Terapia Intensiva Geral e Enfermaria Covid.

Farão parte da pesquisa aqueles profissionais que atenderão aos critérios de inclusão, a saber: ser enfermeiro com vínculo empregatício com a Instituição hospitalar; estar lotado em uma das unidades de assistência ao paciente com Covid-19, ser maior de idade e consentir participar do estudo de maneira voluntária assinando o Termo de Consentimento Livre e Esclarecido. Excluir-se-ão os profissionais em período de férias, licença motivos de saúde ou ausente do setor após três tentativas de contato no decorrer da coleta de dados.

Como instrumento de coleta de dados será realizada uma entrevista semiestruturada. Entrevista semiestruturada é entendida como um espaço relacional de diálogo a respeito de um tema específico, que vai em busca dos significados atribuídos à experiência humana. ${ }^{9}$ Realizar-se-á a entrevista presencialmente pelos pesquisadores nos períodos de turno de trabalho (diurno e noturno) dos colaboradores da Instituição. As mesmas serão gravadas para guardar a fidedignidade das falas dos sujeitos participantes e em ocasião oportuna será realizada a transcrição na integra.

De porte das informações os dados serão analisados conforme categorias. Categoria, em geral, se refere a um conceito que abrange elementos ou aspectos com características comuns ou que se relacionam entre si. Essa palavra está ligada à ideia de classe ou série. As categorias são empregadas para se estabelecer classificações. Nesse sentido, trabalhar com elas significa agrupar elementos, ideias ou expressões em torno de um conceito capaz de abranger tudo isso. ${ }^{8}$ Essa estrutura é muito presente na técnica de análise de dados qualitativa.

Esta pesquisa obedecerá aos princípios éticos estabelecidos na Resolução $n^{\circ} 466 / 12$ do Conselho Nacional de Saúde. ${ }^{10}$ A mesma foi encaminhada ao Comitê de Ética e Pesquisa em Seres Humanos por meio da plataforma Brasil e obteve parecer favorável sob n. CAAE 33496120.1.0000.8088.

\section{Resultados Esperados}

Com o estudo espera-se revelar as vivências dos enfermeiros assistenciais atuantes na linha de frente durante a pandemia pelo novo coronavírus. Por meio deste, possibilitará entender seus sentimentos, medos, anseios e se tornar instrumento de pesquisa para subsidiar ações de prevenção às doenças emocionais.

\section{Referências}

1. World Health Organization (WHO). Coronavírus disease (COVID19): weekly epidemiological update. 2020. Disponível em: https://www.who. int/emergencies/diseases/novel-coronavirus-2019/question-and-answershub/q-a-detail/q-a-coronaviruses\#: :text=symptoms.

2. Zhan Y, Wei L, LI H, Pan Y, Wang JLI, Q et al. The process of psychological change of frontline nurses who care for patientes with COVID19 during their outbreak. Precision Clinical Medicine, 2020;00(00):1-6. https://www.ncbi.nlm.nih.gov/pmc/articles/PMC7107095/pdf/pbaa006.pdf

3. Magrani GC, Bandeira LLB, Santos SCM, Silva CPO, Trajano ETL, Aragão IPB. Relato de experiência de um serviço de emergência do interior do Rio de Janeiro com a pandemia de COVID-19. Revista Pró-UniverSUS. 2021 Jan./Jun.; 12 (1): 14-18.

4. Teixeira CFS, Soares CM, Souza EA, Lisboa ES, Pinto ICM, Andrade LR et al. A saúde dos profissionais de saúde no enfrentamento da pandemia de Covid-19. Ciênc. saúde coletiva. 2020; 25(9), 3465-3474.

5. Santos WA, et al. O impacto da pandemia da COVID-19 na saúde mental dos profissionais de saúde: revisão integrativa. RSD. 2020; 9(8).

6. Barbosa DJ, Gomes MP, Souza FBA, Gomes AMT. Fatores de estresse nos profissionais de enfermagem no combate à pandemia da COVID-19: Síntese de Evidências. Com. Ciências Saúde. 2020; 31(suppl 1):31-47.

7. Migueis GS et al. Condições de trabalho autorreferida por profissionais de saúde durante a pandemia da Covid-19: Revisão integrativa. Research, Society and Development. 2021; 10(6).

8. Minayo MCS (org.). Pesquisa Social. Teoria, método e criatividade. $18^{\mathrm{a}}$ ed. Petrópolis: Vozes, 2001.

9. Moré CLOO. A "entrevista em profundidade" ou "semiestruturada", no contexto da saúde - Dilemas epistemológicos e desafios de sua construção e aplicação. Investigação Qualitativa em Ciências Sociais. 2015; vol.3, Atas CIAIQ.

10. Brasil, Ministério da Saúde. Conselho Nacional de Saúde. Resolução $\mathrm{n}^{\circ} 466$, de 12 de dezembro de 2012. Aprova normas regulamentadoras de pesquisas envolvendo seres humanos. Brasília: Ministério da Saúde. 2013. 